\title{
Endobronchial ultrasound-guided transbronchial needle aspiration of lymph nodes in the radiologically normal
} mediastinum

\author{
F.J.F. Herth*, A. Ernst*”, R. Eberhardt*, P. Vilmann`, H. Dienemann ${ }^{+}$and M. Krasnik $^{\S}$
}

ABSTRACT: Endobronchial ultrasound-guided transbronchial needle aspiration (EBUS-TBNA) can sample enlarged mediastinal lymph nodes in patients with nonsmall cell lung cancer (NSCLC). To date, EBUS-TBNA has only been used to sample nodes visible on computed tomography (CT). The aim of the present study was to determine the accuracy of EBUS-TBNA in sampling nodes $\leqslant 1 \mathrm{~cm}$ in diameter.

NSCLC patients with CT scans showing no enlarged lymph nodes (no node $>1 \mathrm{~cm}$ ) in the mediastinum underwent EBUS-TBNA. Identifiable lymph nodes at locations 2r, 2l, 4r, 4l, 7, 10r, 10l, $11 \mathrm{r}$ and $11 \mathrm{l}$ were aspirated. All patients underwent subsequent surgical staging. Diagnoses based on aspiration results were compared with those based on surgical results.

In 100 patients (mean age 58.9 yrs; 68 males), 119 lymph nodes ranging 5-10 $\mathrm{mm}$ in size were detected and sampled. Malignancy was detected in 19 patients but missed in two; all diagnoses were confirmed by surgical findings. The mean diameter of the punctured lymph nodes was $8.1 \mathrm{~mm}$. The sensitivity of EBUS-TBNA for detecting malignancy was $92.3 \%$, specificity was $100 \%$, and the negative predictive value was $96.3 \%$. No complications occurred.

In conclusion, endobronchial ultrasound-guided transbronchial needle aspiration can accurately sample even small mediastinal nodes, therefore avoiding unnecessary surgical exploration in one out of six patients who have no computed tomography evidence of mediastinal disease. Potentially operable patients with no signs of mediastinal involvement on computed tomography may benefit from pre-surgical endobronchial ultrasound-guided transbronchial needle aspiration and staging.

KEYWORDS: Endobronchial ultrasound, lung cancer, mediastinal lymphadenopathy, transbronchial needle aspiration

$\mathbf{T}$ he staging of lung cancer [1,2] not only provides important information with regard to survival, but also guides treatment. For patients whose disease has metastasised to mediastinal lymph nodes (stage III) or with tumours that have invaded mediastinal structures, the benefit of surgery as primary therapy is questionable. Combined chemoradiotherapy is most appropriate, although chemoradiotherapy followed by surgery may be considered [2]. Mediastinal lymph node involvement is found in $26 \%$ of newly diagnosed lung cancer patients, and extrathoracic metastases are found in $49 \%$ [2]. Thus, reliable staging of mediastinal lymph nodes and the mediastinum is essential for choosing appropriate therapy.
In most centres, computed tomography (CT) is the initial method for staging mediastinal nodes. Nodes detectable by CT that are considered abnormal generally have a short-axis diameter $>1 \mathrm{~cm}$. Smaller lymph nodes can harbour metastatic foci, and enlarged nodes may be benign, especially when central tumours are accompanied by inflammation. Therefore, the accuracy of CT for diagnosing mediastinal disease is low. In a recent meta-analysis of 20 studies and 3,829 patients [3], the pooled sensitivity was $57 \%$, the pooled specificity was $82 \%$ and the pooled negative predictive value was $82 \%$ (range 63$85 \%$ ). Therefore, surgical mediastinal staging is commonly performed in patients with a radiologically normal mediastinum before a planned
AFFILIATIONS

Depts of *Pneumology and Critical Care Medicine, and

${ }^{+}$Thoracic Surgery, Thoraxklinik at the University of Heidelberg, Heidelberg, Germany.

\#Interventional Pulmonology, Beth Israel Deaconess Medical Center, Harvard Medical School, Boston, MA USA.

Depts of "Surgical Gastroenterology, and

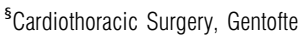
University Hospital, Copenhagen, Denmark.

CORRESPONDENCE

F.J.F. Herth

Dept of Internal Medicine,

Pneumology and Critical Care

Medicine

Thoraxklinik Heidelberg

Amalienstr. 5

D-69126 Heidelberg

Germany

Fax: 4962213961202

E-mail: Felix.Herth@thoraxklinik-

heidelberg.de

Received:

October 262005

Accepted after revision:

June 082006

European Respiratory Journal

Print ISSN 0903-1936

Online ISSN 1399-3003 
cancer resection, to rule out unexpected N2 or N3 disease. Approximately $18 \%$ (range $15-37 \%$ ) of patients with a negative CT scan who undergo surgical mediastinal staging are found to have metastatic disease.

Mediastinoscopy remains the reference standard for evaluating nodal disease. It has a sensitivity of 90-95\% [4]. However, only certain mediastinal lymph node stations are accessible (levels 2, 4, and anterior level 7). For sampling levels 5 and 6, thoracoscopy, anterior mediastinotomy (the Chamberlain procedure), or extended mediastinoscopy can be performed. The inferior mediastinum may be evaluated by thoracoscopy. However, all these more aggressive staging procedures require general anaesthesia, surgical incision, and, therefore, are associated with considerable costs $[5,6]$. Positron emission tomography (PET) was expected to increase the accuracy of mediastinal staging in nonsmall cell lung cancer (NSCLC), and indeed, a meta-analysis has confirmed its superiority [7]. However, more recent reports have tempered enthusiasm for using PET as the sole tool for evaluating the mediastinum [8].

Endoscopic ultrasound (EUS)-guided fine-needle aspiration has been reported as an endoscopic option to evaluate the mediastinum [9-12]. Unfortunately, it does not allow for airway inspection during the procedure. Several authors [1315] have reported that a real-time procedure, endobronchial ultrasound-guided transbronchial needle aspiration (EBUSTBNA) is a highly accurate and safe method for sampling enlarged mediastinal lymph nodes. However, EBUS-TBNA has not been applied to patients with CT-negative scans. The aim of the present study was to determine the accuracy of EBUSTBNA for mediastinal lymph nodes from NSCLC patients without enlarged mediastinal lymph nodes on chest CT scans.

\section{MATERIALS AND METHODS}

The protocol of this multicentre study was approved by the Institutional Review Boards (Heidelberg, Germany). All patients provided written informed consent. Between January 2003 and March 2005, consecutive patients with an indication for bronchoscopy were screened for the study. A chest radiograph and $\mathrm{CT}$ scan of the chest (plain and contrast enhanced) were performed in all patients. Only patients without CT evidence of enlarged mediastinal lymph nodes were included in the current study.

Standard, conventional flexible bronchoscopy (model BF-T160 bronchoscope; Olympus, Tokyo, Japan) was first performed to examine the tracheobronchial tree, followed by EBUS-TBNA using the ultrasound bronchoscope (model XBF-UC160F-OL8; Olympus). Both bronchoscopy procedures were performed with the patient under local anaesthesia and sedation (midazolam) or under general anaesthesia. All patients underwent surgical staging within 10 days after the EBUS-TBNA.

\section{Noninvasive imaging}

Multislice CT was performed in all patients, and tumour/ node/metastasis (TNM) staging, including identification of distant metastases, was recorded by two thoracic CT radiologists. On-site CT examinations were performed with a Siemens helical scanner (Erlangen, Germany), using a single breathhold technique. Off-site CT scans were evaluated by the same radiologists and were included if their quality was similar to the on-site CT studies. If the quality of an off-site CT was inadequate, an on-site CT was performed. Lymph nodes were considered enlarged if the short-axis diameter was $>1 \mathrm{~cm}$.

\section{EBUS-TNBA}

Endobronchial ultrasound (EBUS) was performed using a linear array ultrasonic bronchoscope (XBF-UC 160F-OL8; Olympus; fig. 1). The instrument is similar to a standard bronchoscope with an outer diameter of $6.9 \mathrm{~mm}$, a $2.0-\mathrm{mm}$ instrument channel, and $30^{\circ}$ oblique forward-viewing optics. An electronic convex array ultrasound transducer is mounted at the distal tip and is covered by a water-inflatable balloon sheath. Scanning is performed at a frequency of 7.5 MHz. The angle of view is $90^{\circ}$, and the direction of view is $30^{\circ}$ forward oblique. Image processing was performed by an Olympus ultrasound processor (EU-C60).

A 22-gauge needle (NA-202C; Olympus) was used to perform transbronchial aspiration. The needle exits the outer covering of the insertion tube at $20^{\circ}$. The needle can be visualised through the optics and on the ultrasound image.

The endoscope was passed through the mouth and vocal cords to the main carina, the balloon, if used, was partially inflated (0.3-0.5 mL water), and the regional lymph node stations of the mediastinum and hilar regions (stations 2, 4, 7, 10 and 11) [16] were systematically imaged and measured (short-axis diameter) during slow withdrawal and rotation of the transducer.

All visualised nodes with a size of 5-10 $\mathrm{mm}$ were punctured. According to the size of normal lymph nodes, nodes $<5 \mathrm{~mm}$ were not punctured [17-19]. Transbronchial needle aspiration was performed under real-time ultrasound control. Needle punctures were performed using the "jabbing" method [20]. Integrated colour power Doppler ultrasound was used to avoid intervening vessels immediately before needle puncture (fig. 2). Every node was punctured twice.

The aspirate was placed onto glass slides, air-dried, stained and classified. Papanicolaou staining [21-23] and light microscopy were performed by a cytopathologist who was blinded

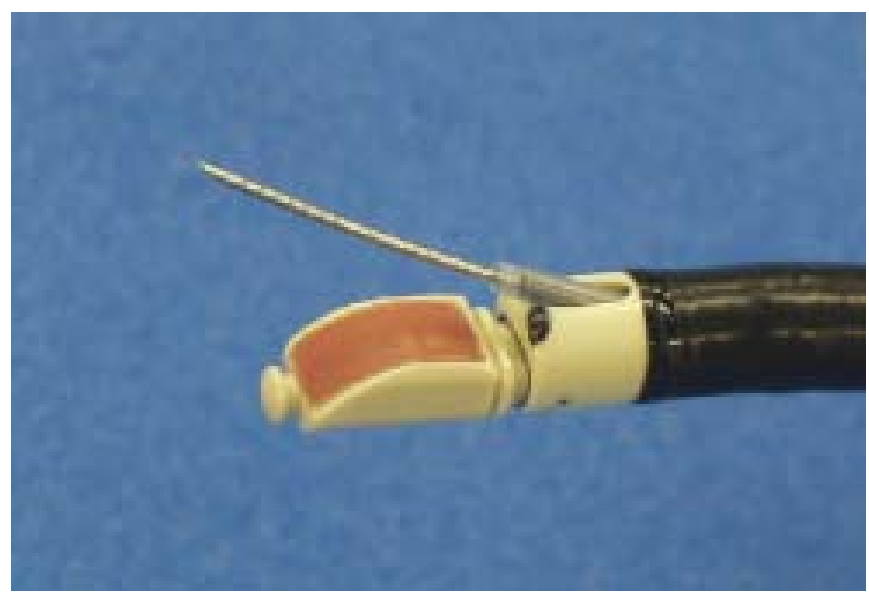

FIGURE 1. Tip of the linear array ultrasonic bronchoscope (BF-UC40F-OL5 Olympus Ltd, Tokyo, Japan). The needle is extended through the working channel and exits obliquely. 


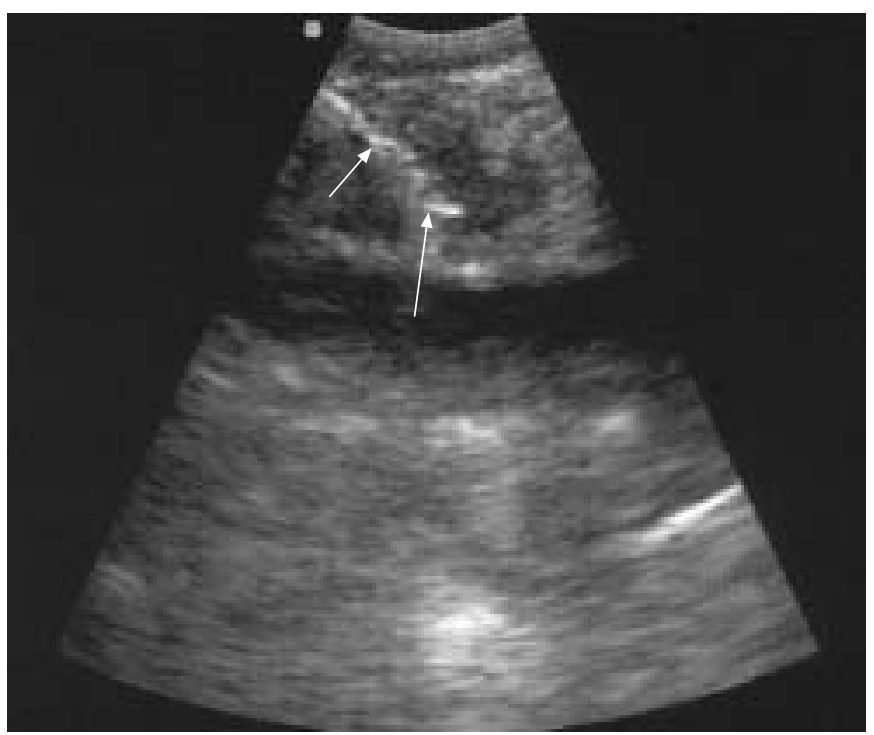

FIGURE 2. Puncture of an 8-mm lymph node in position $4 \mathrm{r}$. The needle is visible (arrows) within the node.

to the details of the patients. No rapid on-site cytology was performed. Diagnoses based on the aspirates were confirmed by open thoracotomy, thoracoscopy or mediastinoscopy in all patients.

\section{Statistical methods}

The Chi-squared test was used, where appropriate, to compare proportional data. The type I error was set at 0.05 for all analyses. Confidence intervals were calculated to $95 \%$ using standard formulae. The sensitivity, specificity and accuracy were calculated using the standard definitions.

\section{RESULTS}

In total, 983 patients were evaluated for lung cancer until 100 were identified (mean age 58.9 yrs; 68 males) with CT evidence suggesting a tumour originating from the lung (T1 to T4), without enlarged mediastinal lymph nodes and a suspicion of or known diagnosis of NSCLC.
A known diagnosis of NSCLC was identified in 87 patients and 13 patients included in the population were highly suspicious for the disease (positive sputum cytology, increased tumour markers, CT criteria and tobacco abuse). All patients had at least one node identified by EBUS and a total of 119 lymph nodes $5-10 \mathrm{~mm}$ in size were detected and punctured by EBUSTBNA (table 1). The mean $\pm S D$ diameter of the punctured lymph nodes was $8.1 \pm 0.7 \mathrm{~mm}$ (range $4-10 \mathrm{~mm}$ ). Additionally, 89 nodes $<5 \mathrm{~mm}$ were detected, which were not punctured. Despite the negative CT scans, EBUS-TBNA of mediastinal lymph nodes was positive for metastases in 19 patients. The stage changed from N0 in three patients to stage N3 disease, to stage N2 disease in 13 patients and to stage N1 disease in three patients. All punctures were adequate and in every smear lymphocytes were visible. In total, 78 patients were examined under general anaesthesia and 22 patients with moderate sedation and topical anaesthesia.

All 100 patients underwent mediastinoscopy (15\%) or thoracotomy (85\%) with mediastinal lymph node resection. Additional positive nodes were detected in two patients, one in the $\mathrm{N} 1$ and one in the N3 position. Overall, 17 patients had stage N2 or N3 disease, of which 16 were identified from EBUS-TBNA, and four patients had stage N1 disease, of which three were identified by EBUS-TBNA. All samples obtained by EBUS-TBNA correctly identified all 79 patients without N1 or $\mathrm{N} 2$ disease. Of the two patients with lymph node metastases not identified by EBUS-TBNA, one had nodal involvement in position 10r and one in position 111, and both had undergone EBUS-TBNA puncture of lymph nodes in these regions. The smears showed lymphocytes, but no malignancy. The sensitivity, specificity and negative predictive value of EBUS-TBNA for detecting malignancy were 92.3, 100 and 96.3\%, respectively.

\section{DISCUSSION}

The current authors found that EBUS-TBNA identified malignant lymph nodes in patients with a normal CT of the mediastinum. The $17 \%$ prevalence of mediastinal lymph node metastases in the present study is similar to that of surgical studies evaluating patients with negative mediastinal CT.

\begin{tabular}{|c|c|c|c|}
\hline Location & Biopsied nodes & Nodes positive for cancer & Surgically confirmed diagnoses \\
\hline $2 r$ & 13 & $5(38)$ & $6(46)$ \\
\hline 21 & 16 & $2(13)$ & $2(13)$ \\
\hline $4 r$ & 17 & $2(12)$ & $2(12)$ \\
\hline $10 \mathrm{r}$ & 12 & $3(25)$ & $3(25)$ \\
\hline 101 & 10 & $1(10)$ & $1(10)$ \\
\hline $11 r$ & 10 & $1(10)$ & $2(20)$ \\
\hline 111 & 11 & $4(36)$ & $4(36)$ \\
\hline Total & 119 & $22(19)$ & $24(20)$ \\
\hline
\end{tabular}


These studies describe metastatic lymph nodes to be present at the time of surgery in 15-20\% of CT-negative patients [4-6].

Compared with mediastinoscopy, EBUS-TBNA has the advantage that it is also able to routinely access posterior mediastinal (level 7) and hilar lymph nodes (levels 10 and 11) and that it can be performed using moderate sedation. Most of the study patients were examined under general anaesthesia, as the examination was done immediately prior to the scheduled surgical procedure. As reported in previous studies, there is no difference in yield or patient tolerance if the procedure is performed under moderate sedation or general anaesthesia [24].

Similar approaches using oesophageal ultrasound (OUS)guided fine-needle aspiration (OUS-FNA) have been described with comparable results, albeit in smaller patient populations. A study by WALLACE et al. [25] demonstrated 69 patients without enlarged mediastinal lymph nodes. OUS detected malignant mediastinal lymph nodes in 14 out of the 69 patients. The sensitivity of EUS for advanced mediastinal disease was $61 \%$ and the specificity was $98 \%$. Additionally, WALLACE et al. [25] found advanced stage in three other patients (one left adrenal metastasis and two with mediastinal invasion of tumour). LEBLANC et al. [26] examined 76 patients with NSCLC without mediastinal lymphadenopathy on CT. EUS-FNA was performed on sites that were suspicious for metastases. Of the 62 patients who underwent surgery, 23 $(37 \%)$ patients had positive lymph nodes, six had peribronchial lymph node (N1) involvement, whereas the remaining 17 patients had ipsilateral or subcarinal lymph node (N2) involvement. Aspirates of lymph nodes yielding lymphocytes only, either by OUS-FNA or EBUS-TBNA, do require followup with a more definitive procedure.

Depending on the lymph node localisation, EBUS-TBNA seems at least comparable to the established EUS-FNA findings in the literature $[15,27,28]$. It is important to note that small lymph nodes are more difficult to identify with any imaging modality (including EBUS) and probably contain a small number of malignant cells, making a cytological diagnosis difficult. Nonetheless, in 100 patients, EBUS-TBNA identified almost all (19 out of 21) patients with advanced disease. The present study supports the theory that EUS-TBNA has excellent potential, even in the patient with a normal mediastinum by CT scanning.

\section{Limitations of the study}

A limitation of the current study was the lack of routine PET scanning. Although PET has become increasingly available and is approved for lung cancer staging, it is still not routinely performed in practice. Recent guidelines for staging of NSCLC recommend PET staging [29], but the evidence supporting this recommendation is only moderate $[7,8]$. Furthermore, the limitations in accuracy discussed above make it unlikely that PET, with its current resolution, even in combination with CT, will replace the need for tissue sampling.

Lymph nodes are often grouped in stations. A potential exists that the surgically sampled lymph nodes in the examined stations were not identical to the ones punctured during endoscopy. This appears unlikely, as in the present routine all lymph nodes are dissected and the results of the endoscopically and surgically sampled lymph nodes are highly congruent.

\section{Conclusions}

The current findings suggest that endobronchial ultrasoundguided transbronchial needle aspiration should be considered in the pre-operative staging of all patients both with and without mediastinal lymph node enlargement on computed tomography scan. Further studies are needed to compare the different invasive and noninvasive staging techniques (computed tomography, positron emission tomography, endobronchial ultrasound-guided transbronchial needle aspiration, endobronchial ultrasound-guided fine-needle aspiration, mediastinoscopy and thoracoscopy) in patients with nonsmall cell lung cancer. To establish a definitive way of staging the mediastinum in patients with nonsmall cell lung cancer, a comparison study of all imaging, minimally invasive and also invasive techniques should be considered.

\section{REFERENCES}

1 Sihoe AD, Yim AP. Lung cancer staging. J Surg Res 2004; 117: 92-106.

2 Spira A, Ettinger DS. Multidisciplinary management of lung cancer. N Engl J Med 2004; 350: 379-392.

3 Toloza EM, Harpole L, Detterbeck F, McCrory DC. Invasive staging of non-small cell lung cancer: a review of the current evidence. Chest 2003; 123: 157-166.

4 Hoffmann H. Invasive staging of lung cancer by mediastinoscopy and video-assisted thoracoscopy. Lung Cancer 2001; 34: 3-5.

5 Luke WP, Pearson FG, Todd TR, Patterson GA, Cooper JD. Prospective evaluation of mediastinoscopy for assessment of carcinoma of the lung. J Thorac Cardiovasc Surg 1986; 91: 53-56.

6 Coughlin M, Deslauriers J, Beaulieu M, et al. Role of mediastinoscopy in pretreatment staging of patients with primary lung cancer. Ann Thorac Surg 1985; 40: 556-560.

7 Toloza EM, Harpole L, McCrory DC. Noninvasive staging of non-small cell lung cancer: a review of the current evidence. Chest 2003; 123: Suppl. 1, 137-146.

8 Brim O, Kappetein AP, Stijnen T, Bogers AJ. Meta-analysis of positron emission tomographic and computed tomographic imaging in detecting mediastinal lymph node metastases in nonsmall cell lung cancer. Ann Thorac Surg 2005; 79: 375-382.

9 Jacobson BC, Hirota WK, Goldstein JL, et al. The role of EUS for evaluation of mediastinal adenopathy. Gastrointest Endoscopy 2003; 58: 819-821.

10 Wallace MB, Woodward TA, Raimondo M. Endoscopic ultrasound and staging of non-small cell lung cancer. Gastrointest Endosc Clin N Am 2005; 5: 157-167.

11 Annema JT, Versteegh MI, Veselic M, Voigt P, Rabe KF. Endoscopic ultrasound-guided fine-needle aspiration in the diagnosis and staging of lung cancer and its impact on surgical staging. J Clin Oncol 2005; 23: 8357-8361.

12 Annema JT, Versteegh MI, Veselic M, et al. Endoscopic ultrasound added to mediastinoscopy for preoperative staging of patients with lung cancer. JAMA 2005; 294: 931-936.

13 Krasnik M, Vilmann P, Larsen SS, Jacobsen GK. Preliminary experience with a new method of endoscopic transbronchial real time ultrasound guided biopsy for 
diagnosis of mediastinal and hilar lesions. Thorax 2003; 58 : 1083-1088.

14 Yasufuku K, Chiyo M, Sekine Y, et al. Real-time endobronchial ultrasound-guided transbronchial needle aspiration of mediastinal and hilar lymph nodes. Chest 2004; 126: 122-128.

15 Rintoul RC, Skwarski KM, Murchison JT, Wallace WA, Walker WS, Penman ID. Endobronchial and endoscopic ultrasound-guided real-time fine-needle aspiration for mediastinal staging. Eur Respir J 2005; 25: 416-421.

16 Mountain CF, Dresler CM. Regional lymph node classification for lung cancer staging. Chest 1997; 111: 17181723.

17 Arita T, Matsumoto T, Kuramitsu T, et al. Is it possible to differentiate malignant mediastinal nodes from benign nodes by size? Reevaluation by $\mathrm{CT}$, transesophageal echocardiography, and nodal specimen. Chest 1996; 110: 1004-1008.

18 Gross BH, Glazer GM, Orringer MB, Spizarny DL, Flint A. Bronchogenic carcinoma metastatic to normal-sized lymph nodes: frequency and significance. Radiology 1988; 166: 71-74.

19 Kerr KM, Lamb D, Wathen CG, Walker WS, Douglas NJ. Pathological assessment of mediastinal lymph nodes in lung cancer: implications for non-invasive mediastinal staging. Thorax 1992; 47: 337-341.

20 Mehta AC, Kavuru MS, Meeker DP, Gephardt GN, Nunez C. Transbronchial needle aspiration for histology specimens. Chest 1989; 96: 1268-1272.

21 Anderson WA, Gunn SA. Cytologic detection of cancer-considerations for its future: a comparative examination of the Papanicolaou and acridine-orange technics. Acta Cytol 1962; 6: 468-470.
22 Herth FJ, Becker HD, Ernst A. Ultrasound-guided transbronchial needle aspiration: an experience in 242 patients. Chest 2003; 123: 604-607.

23 Okamoto H, Watanabe K, Nagatomo A, et al. Endobronchial ultrasonography for mediastinal and hilar lymph node metastases of lung cancer. Chest 2002; 121: 1498-1506.

24 Herth FJF, Becker HD, Ernst A. Conventional versus endobronchial ultrasound-guided transbronchial needle aspiration: a randomized trial. Chest 2004; 125: 322-325.

25 Wallace MB, Ravenel J, Block MI, et al. Endoscopic ultrasound in lung cancer patients with a normal mediastinum on computed tomography. Ann Thorac Surg 2004; 77: 1763-1768.

26 LeBlanc JK, Devereaux BM, Imperiale TF, et al. Endoscopic ultrasound in non-small cell lung cancer and negative mediastinum on computed tomography. Am J Respir Crit Care Med 2005; 171: 177-182.

27 Vilmann P, Krasnik M, Larsen SS, Jacobsen GK, Clementsen P. Transesophageal endoscopic ultrasoundguided fine-needle aspiration (EUS-FNA) and endobronchial ultrasound-guided transbronchial needle aspiration (EBUS-TBNA) biopsy: a combined approach in the evaluation of mediastinal lesions. Endoscopy 2005; 37: 833-839.

28 Herth FJ, Lunn W, Eberhardt R, Becker HD, Ernst A. Transbronchial versus transesophageal ultrasound-guided aspiration of enlarged mediastinal lymph nodes. Am J Respir Crit Care Med 2005; 171: 1164-1167.

29 Pfister DG, Johnson DH, Azzol CG, et al. American Society of Clinical Oncology treatment of unresectable non-smallcell lung cancer guideline: update 2003. J Clin Oncol 2003; 22: 330-353. 